\title{
BMJ Open Association of biological antirheumatic therapy with risk for type 2 diabetes: a retrospective cohort study in incident rheumatoid arthritis
}

\author{
Sanjoy K Paul (D) , ${ }^{1}$ Olga Montvida, ${ }^{1}$ Jennie H Best, ${ }^{2}$ Sara Gale, ${ }^{2}$ \\ Attila Pethö-Schramm, ${ }^{3}$ Khaled Sarsour ${ }^{2}$
}

To cite: Paul SK, Montvida 0, Best JH, et al. Association of biological antirheumatic therapy with risk for type 2 diabetes: a retrospective cohort study in incident rheumatoid arthritis. BMJ Open 2021;11:e042246. doi:10.1136/ bmjopen-2020-042246

- Prepublication history and additional material for this paper are available online. To view these files, please visit the journal online (http://dx.doi org/10.1136/bmjopen-2020042246).

Received 29 June 2020 Revised 26 February 2021 Accepted 04 March 2021

Check for updates

(c) Author(s) (or their employer(s)) 2021. Re-use permitted under CC BY-NC. No commercial re-use. See rights and permissions. Published by BMJ.

${ }^{1}$ Melbourne EpiCentre, University of Melbourne and Melbourne Health, Parkville, Victoria, Australia

${ }^{2}$ Pharmaceuticals Division, Genentech, South San Francisco, California, USA

${ }^{3}$ Pharmaceuticals Division, $F$ Hoffmann-La Roche AG, Basel, Basel-Stadt, Switzerland

Correspondence to

Dr Sanjoy K Paul;

sambhupaul@hotmail.com

\section{ABSTRACT}

Objective To explore possible associations of treatment with biological disease-modifying antirheumatic drugs (bDMARDs), including T-cell-based and interleukin-6 inhibition (IL-6i)-based therapies, and the risk for type 2 diabetes mellitus (T2DM) in patients with rheumatoid arthritis (RA).

Study design, setting and participants Five treatment groups were selected from a United States Electronic Medical Records database of 283756 patients with RA (mean follow-up, 5 years): never received bDMARD (No bDMARD, $n=125337$ ), tumour necrosis factor inhibitors (TNFi, $n=34$ 873), IL-6i $(n=1884)$, T-cell inhibitors $(\mathrm{n}=5935)$ and IL-6i+T cell inhibitor abatacept $(\mathrm{n}=1213)$. Probability and risk for T2DM were estimated with adjustment for relevant confounders.

Results In the cohort of 169242 patients with a mean 4.5 years of follow-up and a mean 641200 person years of follow-up, the adjusted probability of developing T2DM was significantly lower in the IL-6i (probability, 1\%; $95 \%$ $\mathrm{Cl} 0.6$ to 2.0), T-cell inhibitor (probability, 3\%; 95\% Cl 2.3 to 3.3 ) and IL-6i+T cell inhibitor (probability, $2 \% ; 95 \% \mathrm{Cl}$ 0.1 to 2.9 ) groups than in the No bDMARD (probability, $5 \%$; $95 \% \mathrm{Cl} 4.6$ to 4.9 ) and TNFi (probability, $4 \%$; $95 \% \mathrm{Cl} 3.7$ to 4.7 ) groups. Compared with No bDMARD, the IL-6i and IL-6i+T cell inhibitor groups had $37 \%(95 \% \mathrm{Cl}$ of HR 0.42 to 0.96$)$ and $34 \%(95 \% \mathrm{Cl}$ of HR 0.46 to 0.93$)$ significantly lower risk for T2DM, respectively; there was no significant difference in risk in the TNFi (HR 0.99; 95\% $\mathrm{Cl} 0.93$ to 1.06 ) and T-cell inhibitor (HR 0.96; $95 \% \mathrm{Cl} 0.82$ to 1.12 ) groups.

Conclusions Treatment with IL-6i, with or without T-cell inhibitors, was associated with reduced risk for T2DM compared with TNFi or No bDMARDs; a less pronounced association was observed for the T-cell inhibitor abatacept.

\section{INTRODUCTION}

Cardiovascular morbidity is the main cause of complications and death in patients with rheumatoid arthritis (RA). ${ }^{1}{ }^{2}$ Although disease activity and chronic inflammation contribute to the burden of cardiovascular disease (CVD), traditional cardiovascular
Strengths and limitations of this study

- Observational studies have reported an association between treatment with a T-cell costimulatory blocker or tumour necrosis factor inhibitor and reduced risk for type 2 diabetes (T2DM) in patients with established rheumatoid arthritis; however, no holistic evaluation of population-level data has been conducted to evaluate the potential association of interleukin-6 (IL-6)-based therapies with the risk for T2DM with or without interactions with other biologics or the risk for T2DM with no biological therapy.

- The novelties of this pharmacoepidemiological study include thorough evaluation of approximately 170000 US patients with rheumatoid arthritis over a mean of 4.5 years of follow-up from nationally representative electronic medical records (EMR), extensive published methodological development to support the robustness of longitudinal data extraction and study cohort identification, use of a new-user design, holistic evaluation of the association of treatment with different classes of biological and non-biological therapies and their possible interactions with the risk for T2DM, comparative assessment of independent or residual benefits of treatment with an IL-6 receptor inhibitor and a T-cell inhibitor, a robust approach to extract the potential roles of confounders and risk factor heterogeneity and investigation of population-level risk factors and comorbidities at the time of RA diagnosis.

- Study limitations include non-availability of or incomplete data for medication adherence, disease activity, pain scores, insurance type, socioeconomic status, diet and physical activity, although potential residual confounding and indication bias remain a common problem in any EMR-based outcome study.

risk factors, including diabetes, should be carefully monitored in these patients.

Cardiovascular comorbidities including type 2 diabetes (T2DM) have been associated with disease activity score (DAS) in people with RA, with a close association between uncontrolled DAS and glucose 
metabolism, whereas a multinational data-based study suggests $14 \%$ prevalence of diabetes in people with RA. $^{3-6}$ It has been shown that inflammatory cytokines, especially tumour necrosis factor $\alpha$ (TNF $\alpha)$, interleukin-1 (IL-1) and interleukin-6 (IL-6), affect insulin and glucose metabolism. ${ }^{7-11}$ Insulin resistance in patients with RA may be related to increased IL-6 levels. ${ }^{12}$ Disease-modifying antirheumatic drugs (DMARDs) can also alter inflammation-associated insulin resistance in patients with RA. ${ }^{171314}$

Treatment with hydroxychloroquine is associated with reduced risk for T2DM, and treatment with glucocorticoids is associated with increased risk for T2DM. ${ }^{8}{ }^{15-18}$ Several studies report decreased risk for T2DM in patients treated with TNF inhibitors (TNFi). ${ }^{19-21}$ Use of other biological DMARDs (bDMARDs), such as anakinra (IL-1 receptor antagonist), has been shown to improve glycaemic profiles in patients with T2DM. ${ }^{22}$ Furthermore, one study of patients with immunological disease without diabetes suggests that inhibition of IL-6 signalling improves insulin sensitivity, thus implying that elevated IL-6 levels in T2DM might be causally involved in the pathogenesis of insulin resistance.$^{23} \mathrm{~A}$ recent study by Ozen $e t a l^{21}$ indicated that treatment with the T-cell costimulatory blocker abatacept is associated with decreased risk for T2DM; this study in approximately 13700 patients with RA reported a $48 \%$ risk reduction for T2DM among those receiving abatacept compared with those receiving methotrexate monotherapy. Based on claims data from the USA, two recent studies evaluated the cardiovascular safety of abatacept or tocilizumab versus TNFi in patients with RA. ${ }^{24}$ The population-level evidence of possible additional benefits of treatment with IL-6 inhibitor (IL-6i)-based therapies, with or without interactions with other biological therapies in terms of overall cardiovascular risk reduction including diabetes, would be of great importance for the prevention and management of chronic disease. However, we are not aware of any observational study that has evaluated the role of TNFi and IL-6i, with or without T-cell therapy, on insulin resistance in patients with incident $\mathrm{RA}$.

The primary aim of this retrospective pharmacoepidemiological outcomes study was to evaluate-with the use of a large, nationally representative, longitudinal, patient-level cohort of electronic medical records (EMRs) from the USA-the possible association of treatment with TNFi, T-cell inhibitor and IL-6i compared with no exposure to any bDMARD (No bDMARD) with the risk for T2DM. Given recent results showing that treatment with abatacept was associated with decreased risk for T2DM ${ }^{21}$ we also aimed to assess the potential role of T-cell costimulatory blockade in the development of T2DM, with or without concomitant IL-6i use.

\section{METHODS}

\section{Data source}

The Centricity Electronic Medical Records (CEMR) incorporate patient-level data from more than 40000 independent physician practices, academic medical centres, hospitals and large integrated delivery networks covering all states of the USA. The similarities in general population characteristics, cardiometabolic conditions and risk factors between the CEMR database and those reported in the US national health surveys have been reported by our research group and others. ${ }^{26} 27$

The database has been used extensively for academic research 262829 and has enabled our research group to report robust methodology for extraction and assessment of longitudinal patient-level medication data ${ }^{30}$ and to gather a detailed account of therapy use in the US population with T2DM. ${ }^{31}$ Clinically driven machine learningbased algorithms to identify patients with T2DM from EMRs have been described by our research group and others. ${ }^{32} 33$

Longitudinal EMRs were available for more than 34 million persons from 1995 to April 2016, with comprehensive patient-level information on demographics and anthropometric, clinical and laboratory variables. Medication data included brand names and doses for medications prescribed, along with start/stop dates and specific fields to track treatment alterations. The CEMR database also contains patient-reported medications, including prescriptions received outside the EMR network and over-the-counter medications.

\section{Study design and patient population}

The study cohort included patients who met the following criteria: (1) RA diagnosis between 1 January 2000 and 30 April 2016, (2) age at diagnosis $\geq 18$ and $\leq 80$ years, (3) complete data on age and sex, (4) RA diagnosis preceded diabetes diagnosis and (5) follow-up for $\geq 6$ months. Because of issues with the quality and completeness of electronic data, only RA diagnoses after 1999 were considered.

Patients with RA were identified by International Classification of Disease Codes (ICD) as follows: ICD9-446.5, 710.0-710.4, 714.0-714.2, 714.8 and 725.x; ICD-10CM-M05.x, M06.x, M31.5, M32.x-M34.x, M35.1, M35.3 and M36.0. Patients with diagnoses of T2DM were identified with the use of a robust machine learning algorithm, which uses a combination of ICD codes, antidiabetes medications and lifestyle-modification interventions. ${ }^{32}{ }^{33}$ Validation of the final terms was conducted until consensus was achieved between data scientists and invited experts in the field. The final cohort was validated in terms of True Positive/Negative Rate and area under receiver operating characteristic curve among other candidates. The general framework for generating code lists is provided in online supplemental figure S1. Patients in whom type 1 diabetes or gestational diabetes developed during follow-up were excluded. CVD was defined as ischaemic heart disease (including myocardial infarction), peripheral vascular arterial disease, heart failure or stroke. Cancer was defined as any malignant neoplasm or carcinoma in situ, excluding melanoma. Anaemia was defined by clinical diagnosis or haemoglobin level $<12.0$ for women and $<13.5 \mathrm{~g} / \mathrm{dL}$ for men 
at diagnosis of RA. Hypertension was defined by clinical diagnosis or systolic blood pressure (SBP) $>140 \mathrm{~mm} \mathrm{Hg}$ at diagnosis of RA.

Demographic variables included sex, age and ethnicity. Measures of body weight, body mass index (BMI), SBP, HbA1c and lipids were calculated as the average of available measurements within a 3-month window of the index date.

Text mining was performed on original medication names to determine the defined generic names and corresponding brand names approved by the US Food and Drug Administration (including drug combinations). The following non-biological DMARDs (nbDMARDs) were identified: methotrexate, sulphasalazine, leflunomide, hydroxychloroquine, chloroquine, minocycline, tofacitinib citrate, azathioprine, cyclophosphamide, penicillamine, cyclosporine, auranofin and mycophenolate (mofetil or sodium). TNFi included adalimumab, certolizumab pegol, etanercept, golimumab and infliximab; IL-6i included tocilizumab; and T-cell costimulatory blocker included abatacept. All generic names listed under the M01A class of Anatomical Therapeutic Chemical (ATC) classification system and acetylsalicylic acid (aspirin; ATC code B01AC06) were considered non-steroidal anti-inflammatory drugs (NSAIDs). ATC codes $\mathrm{H} 02$ and N02A were used to obtain generic names for corticosteroids and opioids, respectively.

In the study cohort, the following mutually exclusive treatment groups were defined: No bDMARD, patients who never received any bDMARD; TNFi, patients who were prescribed any TNFi and were never exposed to IL-6i or T-cell costimulatory blocker; IL-6i, patients who were exposed to IL-6i but not to T-cell costimulatory blocker at any time during follow-up; T-cell, patients who were exposed to T-cell costimulatory blocker but not to IL-6i at any time during follow-up; IL-6i+T cell, patients who were exposed to IL-6i and T-cell costimulatory blocker at any time during follow-up.

Index date (baseline) was defined as the date of the first prescription of each drug within each group on or after the diagnosis of RA (new-user design). Extensive data mining was conducted to evaluate whether patients were exposed to any RA therapy of interest before the clinical diagnosis of RA. Patients who received any DMARD before the diagnosis of RA were excluded from the study cohort. Time to event for T2DM was defined using the index date and the date of clinical diagnosis of T2DM or was otherwise censored to the last follow-up data in the database. Separate subgroup analyses were conducted in a subcohort of patients with no history of CVD, chronic kidney disease (CKD) or cancer at the time of RA diagnosis (no disease history subcohort). Sensitivity analyses included an exposure-based study design, where the time to development of T2DM was calculated from the time of diagnosis of RA, and an observation of T2DM events after 1 year of follow-up after the index date to address possible undiagnosed T2DM.

\section{Patient and public involvement}

Patients or the public were not involved in the design, or conduct, or reporting, or dissemination plans of our research.

\section{Ethics approval}

This study involved the use of patient-level EMRs, and the patients could not be identified either directly or through linked identifiers. Therefore, according to the US Department of Health and Human Services Exemption 4 (CFR 46.101 (b) (4)), this study is exempt from ethics approval from an institutional review board and from informed consent.

\section{Statistical methods}

Baseline characteristics were summarised as number (\%), mean (SD) or median (first quartile, third quartile) as appropriate. Given the observational nature of the study with heterogeneous baseline characteristics among treatment groups, multinomial propensity scores ${ }^{34}$ were calculated and inverse probability (IP) of exposure weights ${ }^{35} 36$ was used to balance treatment groups on baseline age categories, sex, BMI, and history of CVD, cancer, CKD, hypertension and anaemia. In the balanced data, Cox proportional hazards were used to assess the risk for T2DM development, and the proportionality assumption was evaluated using Schoenfeld testing on individual covariates and for the overall model. Akaike and Bayesian information criteria were used to select the best model fit. The final model was adjusted for age, sex, BMI category (normal, overweight, obese), hypertension, anaemia, statin use, any nbDMARD, methotrexate and hydroxychloroquine. Separate adjustments for other drugs (including corticosteroids and NSAIDs) were assessed but were not included in the final model (based on information criteria assessment). HRs and robust 95\% CIs were reported. Additional sensitivity analysis was conducted to compare HRs from the IP-weighted model and the simple covariate/confounder-adjusted model.

Separate analyses using the treatment effect modellingbased balancing approach, with balancing and adjustments consistent with those described, ${ }^{37}$ were conducted to estimate the probability $(95 \% \mathrm{CI}$ ) of T2DM developing. Additional sensitivity analyses included probability estimation in patients without CVD at baseline. Crude rates $(95 \% \mathrm{CI})$ of T2DM incidence per 1000 person years (PY) were calculated with a standard life-table approach. Although Cox regression-based survival analysis was conducted after propensity matching on the risk factors, baseline data were presented for the original cohort as extracted based on the study design to allow for clear understanding of risk factor distribution at baseline in different therapy groups.

\section{RESULTS}

The study cohort included 125337 patients in the No bDMARD group, 34873 patients in the TNFi group, 1884 patients in the IL-6i group, 5935 patients in the 


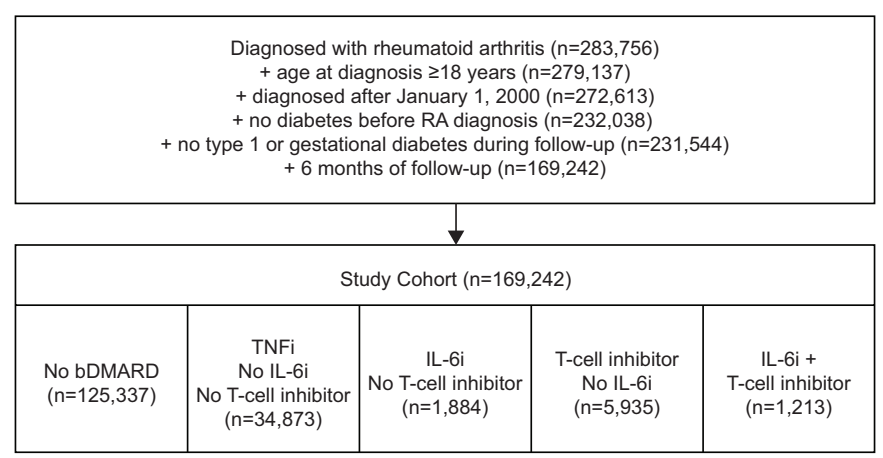

Figure 1 Flow chart of the study cohort. bDMARD, biological disease-modifying antirheumatic drug; IL-6i, interleukin-6 inhibitor; RA, rheumatoid arthritis; TNFi, tumour necrosis factor inhibitor.
T-cell group and 1213 patients in the IL-6i+T cell group (figure 1, table 1). The mean follow-up from RA diagnosis was 4.1 years in the No bDMARD group, 5.4 years in the TNFi group, 4.9 years in the IL-6i group, 5.9 years in the T-cell group and 7.1 years in the IL-6i+T cell group (table 1). The mean age of patients ranged between 52 and 58 years. Higher proportions of patients in the No bDMARD group had a history of hypertension $(40 \%)$ and cancer $(12 \%)$ at or before the RA diagnosis compared with other groups (25\%-28\% hypertension, 4\%-7\% cancer).

In the No bDMARD, TNFi, IL-6i, T-cell and IL-6i+T cell groups, respectively, any nbDMARD was used by $51 \%, 82 \%, 87 \%, 87 \%$ and $93 \%$ of patients; methotrexate was used by $30 \%, 65 \%, 68 \%, 65 \%$ and $74 \%$ of patients; hydroxychloroquine was used by $24 \%, 25 \%, 28 \%, 33 \%$

\section{Table 1 Characteristics of the study cohorts}

\begin{tabular}{|c|c|c|c|c|c|c|}
\hline & No bDMARD & TNFi & IL-6i & T-cell inhibitor & $\begin{array}{l}\text { IL-6i+T cell } \\
\text { inhibitor }\end{array}$ & Total \\
\hline $\mathrm{N}$ & 125337 & 34873 & 1884 & 5935 & 1213 & 169242 \\
\hline Follow-up after index, mean (SD), years & $3.8(3.1)$ & $4.4(3.3)$ & $2.3(1.4)$ & $3.4(2.3)$ & $4.4(2.4)$ & $3.9(3.1)$ \\
\hline Age, mean (SD), years & $58(14)$ & $54(13)$ & $54(13)$ & $55(13)$ & $52(12)$ & $57(14)$ \\
\hline \multicolumn{7}{|l|}{ Age category, years } \\
\hline $40-50$ & $18158(14)$ & $6741(19)$ & $361(19)$ & $1077(18)$ & $310(26)$ & $26647(16)$ \\
\hline $50-60$ & $29316(23)$ & $10162(29)$ & $560(30)$ & $1660(28)$ & $346(29)$ & $42044(25)$ \\
\hline $60-70$ & $33833(27)$ & $8827(25)$ & $457(24)$ & $1559(26)$ & $270(22)$ & $44946(27)$ \\
\hline $70+$ & $29799(24)$ & $3849(11)$ & $213(11)$ & $871(15)$ & $95(8)$ & $34827(21)$ \\
\hline Male & $30696(24)$ & $8451(24)$ & $330(18)$ & $963(16)$ & $189(16)$ & $40629(24)$ \\
\hline \multicolumn{7}{|l|}{ BMI category } \\
\hline Normal & $29502(29)$ & $7873(29)$ & $415(28)$ & $1411(30)$ & $247(26)$ & 39448 (29) \\
\hline Overweight & $33233(33)$ & 8605 (32) & $495(33)$ & $1492(32)$ & $313(32)$ & $44138(32)$ \\
\hline Obese & $39251(38)$ & $10765(40)$ & $596(40)$ & $1800(38)$ & $408(42)$ & 52820 (39) \\
\hline $\begin{array}{l}\text { Systolic blood pressure, mean (SD), } \\
\mathrm{mm} \mathrm{Hg}\end{array}$ & $128(16)$ & $126(16)$ & $124(17)$ & $125(16)$ & $125(16)$ & $127(16)$ \\
\hline Hypertension & $50428(40)$ & $9827(28)$ & $492(26)$ & $1668(28)$ & $299(25)$ & $62714(37)$ \\
\hline HbA1c, mean (SD), \% & $5.8(0.7)$ & $5.6(0.5)$ & $5.6(0.6)$ & $5.7(0.9)$ & $5.7(0.3)$ & $5.8(0.7)$ \\
\hline LDL, mean (SD), mg/dL & $112(35)$ & $113(34)$ & $108(31)$ & $110(36)$ & $116(34)$ & $112(35)$ \\
\hline
\end{tabular}

All values are $n(\%)$ unless stated otherwise.

bDMARD, biological disease-modifying antirheumatic drug; BMI, body mass index; HDL, high-density lipoprotein; IL-6i, interleukin-6 inhibitor; LDL, low-density lipoprotein; Q1, first quartile; Q3, third quartile; RA, rheumatoid arthritis; TNFi, tumour necrosis factor inhibitor. 
Table 2 Medication exposure before type 2 diabetes or end of follow-up

\begin{tabular}{|c|c|c|c|c|c|c|}
\hline & No bDMARD & TNFi & IL-6i & T-cell inhibitor & $\begin{array}{l}\text { IL-6i+T cell } \\
\text { inhibitor }\end{array}$ & Total \\
\hline nbDMARD & $63746(51)$ & 28455 (82) & $1646(87)$ & $5156(87)$ & $1130(93)$ & $100133(59)$ \\
\hline Hydroxychloroquine & $30225(24)$ & $8612(25)$ & $535(28)$ & 1968 (33) & $435(36)$ & $41775(25)$ \\
\hline Anakinra & - & $128(0)$ & $25(1)$ & $43(1)$ & $23(2)$ & $219(0)$ \\
\hline NSAID & $88968(71)$ & $25988(75)$ & $1441(76)$ & $4720(80)$ & $1042(86)$ & $122159(72)$ \\
\hline Statin & 41267 (33) & $10252(29)$ & $621(33)$ & $1961(33)$ & $420(35)$ & $54521(32)$ \\
\hline
\end{tabular}

All values are $n(\%)$.

bDMARD, biological disease-modifying antirheumatic drug; IL-6i, interleukin-6 inhibitor; nbDMARD, non-biological disease-modifying antirheumatic drug; NSAID, non-steroidal anti-inflammatory drug; TNFi, tumour necrosis factor inhibitor.

and $36 \%$ of patients; and statins were used by $33 \%, 29 \%$, $33 \%, 33 \%$ and $35 \%$ of patients (table 2 ).

Over 641200 PY of follow-up, 4.4\%, 5.2\%, 1.7\%, 3.9\% and $3.1 \%$ of patients had clinical diagnoses of T2DM in the No bDMARD, TNFi, IL-6i, T-cell and IL-6i+T cell groups, respectively. The incidence rate/1000 PY of T2DM was significantly lower in the IL-6i $(7.6$; 95\% CI 5.4 to 10.8$)$ and IL-6i+T cell $(7.0 ; 95 \%$ CI 5.1 to 9.7$)$ groups than in the No bDMARD group (12.1; 95\% CI 11.8 to 12.4) (table 3). The incidence rate/1000 PY of T2DM in the TNFi and T-cell groups was similar to that in the No bDMARD group (table 3 ).

The adjusted probability of developing T2DM was significantly lower in the IL-6i (probability, $1 \%$; 95\% CI 0.6 to 2.0 ) and IL-6i+T cell (probability, $2 \%$; $95 \%$ CI 0.1 to 2.9) groups and somewhat less pronounced in the T-cell group (probability, 3\%; 95\% CI 2.3 to 3.3) than in the No bDMARD (probability, 5\%; 95\% CI 4.6 to 4.9 ) and TNFi (probability, 4\%; 95\% CI 3.7 to 4.3 ) groups (table 3).

Sensitivity analyses based on data from patients without CVD at baseline produced similar probability estimates.

Compared with patients in the No bDMARD group, those in the IL-6i and IL-6i+T cell groups had 37\% (95\% CI of HR $0.42 \%$ to $0.96 \%$ ) and $34 \%$ (95\% CI of HR $0.46 \%$ to $0.93 \%$ ) significantly lower risk for T2DM, respectively, though there was no significant difference in estimated risk for patients in the TNFi (95\% CI of HR 0.93 to 1.06) and T-cell (95\% CI of HR 0.82 to 1.12 ) groups (figure 2, table 3). These probability/risk estimates were similar in the no disease history subcohort, the cancer at index subcohort and the exposure-based sensitivity analyses and in the analysis in which any event of T2DM within 1 year of follow-up was excluded.

Table 3 Proportions of patients who developed type 2 diabetes (T2DM), incidence rate (95\% CI) per 1000 person years of T2DM in the study cohorts, adjusted HR $(95 \% \mathrm{CI})$ (based on propensity weights and on without propensity weights using only covariates) and adjusted probability $(95 \% \mathrm{Cl})$ of developing T2DM in the study cohorts (based on new user design)

\begin{tabular}{|c|c|c|c|c|c|}
\hline & $\begin{array}{l}\text { T2DM event } \\
(\%)\end{array}$ & $\begin{array}{l}\text { Incidence rate } \\
(95 \% \mathrm{Cl})\end{array}$ & HR (95\% Cl); p* & HR (95\% Cl); p† & $\begin{array}{l}\text { Adjusted probability } \\
(95 \% \mathrm{Cl})\end{array}$ \\
\hline No bDMARD & $5544(4)$ & 12.1 (11.8 to 12.4$)$ & Reference & Reference & 0.048 (0.046 to 0.049$)$ \\
\hline $\begin{array}{l}\text { IL-6i, no T-cell } \\
\text { inhibitor }\end{array}$ & $32(2)$ & $7.6(5.4$ to 10.8$)$ & $\begin{array}{l}0.63(0.42 \text { to } 0.96) ; \\
0.021\end{array}$ & $\begin{array}{l}0.58(0.40 \text { to } 0.94) \\
0.019\end{array}$ & $0.013(0.006$ to 0.020$)$ \\
\hline $\begin{array}{l}\text { IL-6i and T-cell } \\
\text { inhibitor }\end{array}$ & $37(3)$ & 7.0 (5.1 to 9.7$)$ & $\begin{array}{l}0.66(0.46 \text { to } 0.93) ; \\
0.019\end{array}$ & $\begin{array}{l}0.62(0.41 \text { to } 0.91) \\
0.017\end{array}$ & 0.020 (0.010 to 0.029$)$ \\
\hline
\end{tabular}

Treatment groups were balanced according to baseline age group, body mass index (BMI) and history of hypertension, anaemia, cardiovascular disease, and cancer or chronic kidney disease and were adjusted for age, sex, BMI category, hypertension and anaemia and for exposure to statins, any non-biological disease-modifying antirheumatic drug (nbDMARD), methotrexate and hydroxychloroquine.

${ }^{*}$ Based on propensity weight.

†Based on covariate adjustments without propensity weight.

IL-6i, interleukin-6 inhibitor; TNFi, tumour necrosis factor inhibitor. 


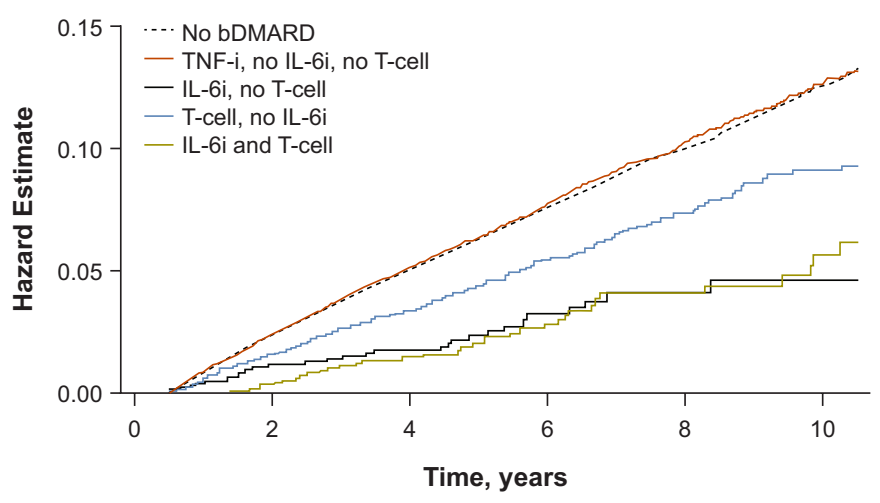

Figure 2 Hazard estimate plot of the adjusted risk for type 2 diabetes. bDMARD, biological disease-modifying antirheumatic drug; IL-6i, interleukin-6 inhibitor; TNFi, tumour necrosis factor inhibitor.

Patients with hypertension had $41 \%$ (95\% CI of HR $1.33 \%$ to $1.49 \%$ ) significantly higher risk for T2DM, and overweight and obese patients had more than twofold and fivefold higher risk for T2DM, respectively. The observed $10 \%-15 \%$ increased risk for T2DM in patients with anaemia was statistically significant in primary and secondary prevention cohorts $(\mathrm{p}<0.01)$. Use of methotrexate combined with hydroxychloroquine was independently associated with $11 \%-12 \%$ (95\% CI of HR $0.82 \%$ to $0.95 \%)$ and $27 \%-30 \%$ (95\% CI of HR $0.65 \%$ to $0.75 \%)$ reduced risk for T2DM in the primary study cohort and in the no disease history subcohort, respectively.

Although statin use (32\% in the cohort) was independently associated with an $11 \%$ (95\% CI of HR $1.05 \%$ to $1.18 \%$ ) increased risk for diabetes in the entire cohort, this association disappeared in the no disease history subcohort. A separate interaction analysis of statin use by age group also consistently revealed insignificant association of statin use with risk for T2DM in the no disease history subcohort (unpublished data).

\section{DISCUSSION}

This EMR-based study from a nationally representative primary and ambulatory care database is novel and includes risk profile assessment at the time of clinical diagnosis of RA and holistic assessment of a potentially beneficial association between treatment with three classes of biologics, including IL-6i signalling blockingbased therapy with and without exposure to a T-cell inhibitor, and risk for T2DM. Comparative assessment of possible independent or residual benefits of treatment with an IL-6 receptor inhibitor and a T-cell inhibitor in patients with long-term diabetes risk adds new insight into the population-level effectiveness of such therapies in the prevention and management of cardiovascular risk in patients with RA.

In this longitudinal cohort study in approximately 170000 patients with incident RA treated with different antirheumatic therapies over a mean 4.5 years of follow-up, we observed the following: compared with no exposure to bDMARDs, exposure to the IL-6 receptor inhibitor tocilizumab was associated with the most pronounced risk reduction for T2DM, followed by a less pronounced association with inhibition of T-cell costimulation with abatacept and no association with TNFi; in patients treated with bDMARDs, exposure to IL-6 receptor inhibitor, with or without T-cell inhibitor, was associated with significantly lower risk for T2DM, and exposure to TNFi and T-cell inhibitor was not independently associated with risk for T2DM; compared with exclusive exposure to the IL-6 receptor inhibitor tocilizumab, additional exposure to the T-cell inhibitor abatacept during therapy did not add any benefit in reducing risk for T2DM.

The observed reduction in risk for T2DM in patients treated with tocilizumab is evident from the difference between hazard estimates 6 months after the initiation of therapy in patients treated with tocilizumab and in the No bDMARD, TNFi and T-cell groups and from the consistently lower risk throughout follow-up (figure 2). The association of IL-6 receptor inhibition with reduced risk for diabetes was consistent in patients with or without a history of major disease. Careful risk analyses, ensuring appropriate balancing for potential confounding and differentiable risk paradigm among comparative treatment groups, allowed reliable inference in this pharmacoepidemiological outcome study with a reasonable follow-up time. IL-6 is overexpressed in insulin resistance and impairs insulin action in liver and adipose tissue. The involvement of IL-6 in the regulation of hepatic insulin sensitivity was highlighted by the neutralisation of IL-6, which showed the subsequent enhancement of hepatic insulin sensitivity. ${ }^{103839}$ Our data suggest a possible proactive therapeutic strategy in improving insulin sensitivity by use of IL- 6 blocking agents in people with RA and prediabetes.

Our observed event rate of 11.7/1000 PY in the T-cell inhibitor group was similar to the reported event rate in the abatacept group (11.4/1000 PY) reported by Ozen et $a .^{21}$ Although the observed $35 \%$ lower event rate/1000 PY in the IL-6i group was not significantly different from that in the T-cell group in our study, the residual association of exposure to IL-6i in terms of reduced risk (34\%$37 \%$ ) for T2DM was evident, with similar follow-up times of 2.3 and 2.6 years after IL-6i initiation in the IL-6i and IL-6i+T cell groups, respectively.

Consistent with previous studies, ${ }^{12} 171821$ we observed that treatment with hydroxychloroquine was independently associated with 27\%-30\% reduced risk for T2DM $(\mathrm{p}<0.01)$; approximately $25 \%$ of patients in the cohort were exposed to hydroxychloroquine during follow-up.

The mean low-density lipoprotein level in our cohort was $112 \mathrm{mg} / \mathrm{dL}$ at the index date. Although approximately $32 \%$ of the cohort was treated with statins, use of this lipid-lowering therapy was not independently associated with risk for T2DM in the primary CVD prevention cohort across all age groups. In our study cohort, approximately $37 \%$ of patients had hypertension; $19 \%$ of those patients had SBP above 140 $\mathrm{mm} \mathrm{Hg}$ at the index date. Patients with hypertension had a 
significant $41 \%$ increased risk for T2DM. A separate interaction assessment suggested that patients who have hypertension and use statins are at marginally higher risk for T2DM than patients who do not have hypertension and are not using statins (HR, 1.34; 95\% CI 1.07 to 1.66 ) and patients who have hypertension and are not using statins (HR, 1.25; 95\% CI 1.06 to 1.38). This finding corroborates the results of a recent population-based study in a large number of Chinese patients with hypertension. ${ }^{40}$ Ozen $e t a l^{21}$ reported a significant increase in the risk for T2DM in approximately $15 \%$ of statin users based on a cohort of 13669 patients with approximately 15 years' duration of RA, though hypertension did not contribute significantly to their models. ${ }^{21}$ Our extensive evaluation of the possible interplay between hypertension and dyslipidaemia in the risk for T2DM in the study cohort also ensures the robustness of the observed association of RA therapies with risk for T2DM.

The CEMR database contains information on medications in the form of prescription dates and number of refills and less frequently captures data on dispensed prescriptions. Although the non-availability of reliable information on medication adherence is a common problem in all clinical studies and the EMRs generally provide only prescription information (not actual pharmacy dispensing information), detailed validation studies of US EMRs suggest a high level of agreement between EMR prescription data and pharmacy claims data, especially in chronic diseases. ${ }^{41}$ However, the availability of data from patients' medication lists, including medications prescribed within the EMR network and medications that could have been prescribed outside the EMR network, is a strength. The CEMR database also tracks longitudinal treatment adjustments. Furthermore, we used robust algorithms to aggregate medication data at the patient level. Finally, the database contains comprehensive clinical information, which is usually not available in claims databases.

There are several potential limitations in any pharmacoepidemiological study based on retrospective EMRs, including incomplete disease coding, missing data (eg, data on prescription dispensation and adherence to therapies). Other limitations with the CEMR database include the non-availability of data on socioeconomic characteristics, diet and physical activity as well as the non-availability of complete or reliable data for disease activity and pain scores, longitudinal data on doses of individual therapies and insurance type. Although the distribution of major cardiometabolic diseases in the CEMR is comparable with the distribution in national survey results, the representativeness of the database in terms of RA has not been formally tested. In addition, patient data moved out of the EMR database generally cannot be tracked. Although we used a carefully drawn new-user design and adjusted for the confounders to the best of data availability, potential residual confounding cannot be completely eliminated from an observational study.

In conclusion, in this large longitudinal cohort study of patients with incident RA, we observed that patients treated with the IL-6 receptor inhibitor tocilizumab had significantly reduced risk for T2DM compared with those treated with other bDMARDs and those not exposed to any bDMARD. This observation also appears to be true for patients treated with bDMARDs in the treatment sequence, irrespective of treatment with T-cell inhibitor before or after IL-6 receptor inhibitor treatment. These findings provide valuable insight for the use of biologics in patients with RA for the overall prevention and management of cardiovascular risk and diabetes risk.

Acknowledgements Melbourne EpiCentre gratefully acknowledges the support from the Australian Government Department of Education's National Collaborative Research Infrastructure Strategy initiative through Therapeutic Innovation Australia.

Contributors All authors were involved in drafting the article or revising it critically for important intellectual content, and all authors approved the final version to be submitted for publication. SKP had full access to all the data in the study and takes responsibility for the integrity of the data and the accuracy of the data analysis. Study conception and design: SKP, KS, OM, JHB, SG, APS. Acquisition of data: OM Analysis and interpretation of data: SKP, OM. Drafted manuscript: SKP.

Funding Roche/Genentech supported manuscript development.

Competing interests SKP has served as a consultant and/or speaker for Novartis, GI Dynamics, Roche, AstraZeneca, Guangzhou Zhongyi Pharmaceutical and Amylin Pharmaceuticals and has received grants in support of investigator and investigator-initiated clinical studies from Merck, Novo Nordisk, AstraZeneca, Hospira, Amylin Pharmaceuticals, Sanofi-Aventis and Pfizer. JHB, SG and KS are employees of Genentech. APS is an employee of F. Hoffmann-La Roche.

Patient consent for publication Not required.

Ethics approval According to the US Department of Health and Human Services Exemption 4 (CFR 46.101(b)(4)), this study is exempt from ethics approval from an institutional review board and from informed consent.

Provenance and peer review Not commissioned; externally peer reviewed.

Data availability statement Data are available upon reasonable request. Qualified researchers may request access to individual patient level data through the clinical study data request platform (https://vivli.org/). Further details on Roche's criteria for eligible studies are available here (https://vivli.org/members/ourmembers/). For further details on Roche's Global Policy on the Sharing of Clinical Information and how to request access to related clinical study documents, see here (https://www. roche.com/research_and_development/who_we_are_how_we_work/clinical_ trials/our_commitment_to_data_sharing.htm).

Supplemental material This content has been supplied by the author(s). It has not been vetted by BMJ Publishing Group Limited (BMJ) and may not have been peer-reviewed. Any opinions or recommendations discussed are solely those of the author(s) and are not endorsed by BMJ. BMJ disclaims all liability and responsibility arising from any reliance placed on the content. Where the content includes any translated material, BMJ does not warrant the accuracy and reliability of the translations (including but not limited to local regulations, clinical guidelines, terminology, drug names and drug dosages), and is not responsible for any error and/or omissions arising from translation and adaptation or otherwise.

Open access This is an open access article distributed in accordance with the Creative Commons Attribution Non Commercial (CC BY-NC 4.0) license, which permits others to distribute, remix, adapt, build upon this work non-commercially, and license their derivative works on different terms, provided the original work is properly cited, appropriate credit is given, any changes made indicated, and the use is non-commercial. See: http://creativecommons.org/licenses/by-nc/4.0/.

ORCID iD

Sanjoy K Paul http://orcid.org/0000-0003-0848-7194

\section{REFERENCES}

1 Ferro F, Elefante E, Luciano N, et al. One year in review 2017: novelties in the treatment of rheumatoid arthritis. Clin Exp Rheumatol 2017;35:721-34. 
2 Semb AG. Handbook of cardiovascular disease management in rheumatoid arthritis. Berlin, Germany: Springer-Verlag, 2017.

3 Dougados M, Soubrier M, Antunez A, et al. Prevalence of comorbidities in rheumatoid arthritis and evaluation of their monitoring: results of an international, cross-sectional study (COMORA). Ann Rheum Dis 2014;73:62-8.

4 Radner $\mathrm{H}$, Yoshida K, Hmamouchi I, et al. Treatment patterns of multimorbid patients with rheumatoid arthritis: results from an international cross-sectional study. J Rheumatol 2015;42:1099-104.

5 Ruscitti P, Ursini F, Cipriani P, et al. Poor clinical response in rheumatoid arthritis is the main risk factor for diabetes development in the short-term: a 1-year, single-centre, longitudinal study. PLoS One 2017;12:e0181203.

6 Donath MY, Shoelson SE. Type 2 diabetes as an inflammatory disease. Nat Rev Immunol 2011;11:98-107.

7 Chung CP, Oeser A, Solus JF, et al. Inflammation-associated insulin resistance: differential effects in rheumatoid arthritis and systemic lupus erythematosus define potential mechanisms. Arthritis Rheum 2008;58:2105-12.

8 Rekedal LR, Massarotti E, Garg R, et al. Changes in glycosylated hemoglobin after initiation of hydroxychloroquine or methotrexate treatment in diabetes patients with rheumatic diseases. Arthritis Rheum 2010;62:3569-73.

9 Suzuki T, Imai J, Yamada T, et al. Interleukin-6 Enhances GlucoseStimulated Insulin Secretion From Pancreatic-Cells: Potential Involvement of the PLC-IP3-Dependent Pathway. Diabetes 2011;60:537-47

10 Ruscitti P, Ursini F, Cipriani P, et al. II-1 inhibition improves insulin resistance and adipokines in rheumatoid arthritis patients with comorbid type 2 diabetes. Medicine 2019;98:e14587-e87.

11 Ruscitti P, Masedu F, Alvaro S, et al. Anti-Interleukin-1 treatment in patients with rheumatoid arthritis and type 2 diabetes (track): a multicentre, open-label, randomised controlled trial. PLoS Med 2019;16:e1002901.

12 Bradham WS, Ormseth MJ, Oeser A, et al. Insulin resistance is associated with increased concentrations of NT-proBNP in rheumatoid arthritis: IL-6 as a potential mediator. Inflammation 2014;37:801-8

13 Baghdadi LR, Woodman RJ, Shanahan EM, et al. The impact of traditional cardiovascular risk factors on cardiovascular outcomes in patients with rheumatoid arthritis: a systematic review and metaanalysis. PLoS One 2015;10:e0117952.

14 Solomon DH, Kremer J, Curtis JR, et al. Explaining the cardiovascular risk associated with rheumatoid arthritis: traditional risk factors versus markers of rheumatoid arthritis severity. Ann Rheum Dis 2010;69:1920-5.

15 Movahedi M, Beauchamp M-E, Abrahamowicz M, et al. Risk of incident diabetes mellitus associated with the dosage and duration of oral glucocorticoid therapy in patients with rheumatoid arthritis. Arthritis Rheumatol 2016;68:1089-98.

16 Wasko MCM, Hubert HB, Lingala VB, et al. Hydroxychloroquine and risk of diabetes in patients with rheumatoid arthritis. JAMA 2007;298:187-93.

17 Sharma TS, Wasko MCM, Tang X, et al. Hydroxychloroquine use is associated with decreased incident cardiovascular events in rheumatoid arthritis patients. J Am Heart Assoc 2016;5:e002867.

18 Xie W, Yang X, Ji L, et al. Incident diabetes associated with hydroxychloroquine, methotrexate, biologics and glucocorticoids in rheumatoid arthritis: a systematic review and meta-analysis. Semin Arthritis Rheum 2020;50:598-607.

19 Antohe JL, Bili A, Sartorius JA, et al. Diabetes mellitus risk in rheumatoid arthritis: reduced incidence with anti-tumor necrosis factor $\alpha$ therapy. Arthritis Care Res 2012;64:215-21.

20 Solomon DH, Massarotti E, Garg R, et al. Association between disease-modifying antirheumatic drugs and diabetes risk in patients with rheumatoid arthritis and psoriasis. JAMA 2011;305:2525-31.

21 Ozen G, Pedro S, Holmqvist ME, et al. Risk of diabetes mellitus associated with disease-modifying antirheumatic drugs and statins in rheumatoid arthritis. Ann Rheum Dis 2017;76:848-54.
22 Ertunc ME, Hotamisligil GS. Lipid signaling and lipotoxicity in metaflammation: indications for metabolic disease pathogenesis and treatment. J Lipid Res 2016;57:2099-114.

23 Schultz O, Oberhauser F, Saech J, et al. Effects of inhibition of interleukin-6 signalling on insulin sensitivity and lipoprotein (a) levels in human subjects with rheumatoid diseases. PLoS One 2010;5:e14328.

24 Kim SC, Solomon DH, Rogers JR, et al. Cardiovascular safety of tocilizumab versus tumor necrosis factor inhibitors in patients with rheumatoid arthritis: a Multi-Database cohort study. Arthritis Rheumatol 2017;69:1154-64.

25 Kang EH, Jin Y, Brill G, et al. Comparative cardiovascular risk of abatacept and tumor necrosis factor inhibitors in patients with rheumatoid arthritis with and without diabetes mellitus: a multidatabase cohort study. J Am Heart Assoc 2018;7:e007393.

26 Brixner D, Said Q, Kirkness C, et al. Assessment of cardiometabolic risk factors in a national primary care electronic health record database. Value Health 2007;10:S29-36.

27 Montvida O, Dibato JE, Paul S. Evaluating the representativeness of US Centricity electronic medical records with reports from the centers for disease control and prevention: comparative study on office visits and cardiometabolic conditions. JMIR Med Inform 2020;8:e17174.

28 Crawford AG, Cote C, Couto J, et al. Comparison of Ge Centricity electronic medical record database and national ambulatory medical care survey findings on the prevalence of major conditions in the United States. Popul Health Manag 2010;13:139-50.

29 Montvida O, Shaw JE, Blonde L, et al. Long-term sustainability of glycaemic achievements with second-line antidiabetic therapies in patients with type 2 diabetes: A real-world study. Diabetes Obes Metab 2018;20:1722-31.

30 Montvida O, Arandjelović O, Reiner E, et al. Data mining approach to estimate the duration of drug therapy from longitudinal electronic medical records. Open Bioinforma J 2017;10:1-15.

31 Montvida O, Shaw J, Atherton JJ, et al. Long-Term trends in Antidiabetes drug usage in the U.S.: real-world evidence in patients newly diagnosed with type 2 diabetes. Diabetes Care 2018;41:69-78.

32 Moreno-Iribas C, Sayon-Orea C, Delfrade J, et al. Validity of type 2 diabetes diagnosis in a population-based electronic health record database. BMC Med Inform Decis Mak 2017;17:34.

33 Owusu Adjah ES, Montvida O, Agbeve J, et al. Data mining approach to identify disease cohorts from primary care electronic medical records: a case of diabetes mellitus. Open Bioinforma $J$ 2017;10:16-27.

34 Ridgeway G, McCaffrey DF, Morral AR. Toolkit for weighting and analysis of nonequivalent groups: a tutorial for the R TWANG package. Santa Monica, CA: Rand Corporation, 2020. https://cran. r-project.org/web/packages/twang/vignettes/twang.pdf

35 Lunceford JK, Davidian M. Stratification and weighting via the propensity score in estimation of causal treatment effects: a comparative study. Stat Med 2004;23:2937-60.

36 McCaffrey DF, Ridgeway G, Morral AR. Propensity score estimation with boosted regression for evaluating causal effects in observationa studies. Psychol Methods 2004;9:403-25.

37 Hlatky MA, Douglas PS, Cook NL, et al. Future directions for cardiovascular disease comparative effectiveness research: report of a workshop sponsored by the National heart, lung, and blood Institute. J Am Coll Cardiol 2012;60:569-80.

38 Klover PJ, Zimmers TA, Koniaris LG, et al. Chronic exposure to interleukin- 6 causes hepatic insulin resistance in mice. Diabetes 2003:52:2784-9.

39 Hoene M, Weigert C. The role of interleukin- 6 in insulin resistance, body fat distribution and energy balance. Obes Rev 2008;9:20-9.

$40 \mathrm{Li} \mathrm{H}$, Lin $\mathrm{H}$, Zhao $\mathrm{H}$, et al. Statins use and risk of new-onset diabetes in hypertensive patients: a population-based retrospective cohort study in Yinzhou district, Ningbo City, people's Republic of China. Ther Clin Risk Manag 2018;14:823-32.

41 Rowan CG, Flory J, Gerhard T, et al. Agreement and validity of electronic health record prescribing data relative to pharmacy claims data: a validation study from a US electronic health record database. Pharmacoepidemiol Drug Saf 2017;26:963-72. 